\title{
TRASFORMAZIONE DI COMBESCURE \\ ED ALTRE ANALOGHE PER LE CURVE STORTE.
}

Nota di Gustavo Sannia, in Torino.

Adunanza del 12 marzo 1905.

L'illustre prof. Branchi ${ }^{*}$ ) ha chiamato trasformazione di Combescure una particolare corrispondenza fra $i$ punti di due curve, e ne ha fatto notevoli applicazioni alla ricerca delle più generali curve a flessione costante, dell'elica sferica, delle traiettorie ortogonali ad una semplice infinità di sfere, delle curve di Bertrand.

Or questa trasformazione, ed altre analoghe, si presentano spontatanee nello studio delle curve per via intrinseca.

Sieno $\rho$ ed $r$ i raggi di prima e seconda curvatura di una curva $C$ in un punto $M, s$ l'arco che ha per termine $M$, computato a partire da un'origine fissa su $C,(x y z)$ le coordinate cartesiane di un punto $M_{1}$, mobile con $M$, rispetto al triedro fondamentale di $C$ in $M$, ossia rispetto alla tangente, alla binormale ed alla normale principale di $C$ in $M$; siano poi $(x+\delta x, y+\delta y, z+\delta z)$ le coordinate del punto $M_{\mathrm{r}}^{\prime}$ in cui si porta $M_{1}$ quando $M$, muovendosi su $C$, passa nel punto infinitamente vicino $M^{\prime}$. Reggono allora le formole fondamentali ${ }^{*}$ )

(I) $\frac{\delta x}{d s}=\frac{d x}{d s}-\frac{z}{\rho}+\mathrm{I}, \frac{\delta y}{d s}=\frac{d y}{d s}-\frac{z}{r}, \frac{\delta z}{d s}=\frac{d z}{d s}+\frac{x}{\rho}+\frac{y}{r}$.

Che se poi $\alpha \beta \gamma$ sono i coseni direttori di una direzione mobile con $M$, si hanno le formole analoghe

(2) $\frac{\delta \alpha}{d s}=\frac{d \alpha}{d s}-\frac{\gamma}{\rho}, \frac{\delta \beta}{d s}=\frac{d \gamma}{d s}-\frac{\gamma}{r}, \frac{\delta \gamma}{d s}=\frac{d \gamma}{d s}+\frac{\alpha}{\rho}+\frac{\beta}{r}$.

") Cfr. Lezioni di Geometria differenziale, $2^{a}$ ed., vol. I, pag. 40 e seg.

**) Cfr. E. Cesàro, Lezioni di Geometria intrinseca, pag. I 24. 
Ponendo nelle (I) $\delta x=\delta y=\delta z=0$, si hanno le relazioni

$$
\frac{d x}{d s}=\frac{z}{p}-\mathrm{I}, \quad \frac{d y}{d s}=\frac{z}{r}, \frac{d z}{d s}=-\frac{x}{\rho}-\frac{y}{r}
$$

necessarie e sufficienti per l'immobilità del punto $M_{\mathrm{r}}$.

Sorge qui spontanea l'idea di annullare due sole delle quantità $\delta x, \delta y, \delta z$.

Ponendo, per esempio, $\delta y=\delta z=0$, il punto $M_{1}$ si muoverd descrivendo una curva $C_{1}$ riferita a $C$ per modo, che nei punti corrispondenti $M$ e $M_{\mathrm{z}}$ esse avranno le tangenti parallele, e però $C_{1}$ è una curva che può dedursi da $C$ con una trasformazione di Combescure.

Evidentensente anche le binormali e le normali principali saranno parallele, e però saranno eguali gli angoli infinitesimi di contingenza e di torsione $\varepsilon$ e $n$; ne segue che,

$$
\frac{\mathrm{I}}{\rho_{\mathrm{I}}}=\frac{\varepsilon}{\delta s_{\mathrm{I}}}=\frac{\varepsilon}{d s} \cdot \frac{d s}{\delta s_{\mathrm{I}}}=\frac{\mathrm{I}}{\rho} \frac{d s}{\delta s_{\mathrm{I}}}, \frac{\mathrm{I}}{r_{\mathrm{I}}}=\frac{\eta}{\delta s_{\mathrm{I}}}=\frac{\eta}{d s} \cdot \frac{d s}{\delta s_{\mathrm{I}}}=\frac{\mathrm{I}}{r} \frac{d s}{\delta s_{\mathrm{I}}},
$$

ove si è dato l'indice $I$ alle quantità che si riferiscono a $C_{t}$, ossia

ove si è posto

$$
p_{1}=p f(s), \quad r_{1}=r f(s),
$$

$$
\frac{\delta s_{\mathrm{r}}}{d s}=f(s) .
$$

Ne segue che una trasformazione di CombesCuRE lascia inalterato il rapporto delle curvature.

Se ne deduce che sono parallele anche le rette rettificanti di $C$ e $C_{1}$ nei punti corrispondenti, perchè l'inclinazione $\theta$ della retta rettificante di $C$ sulla tangente è definita dalla formola ${ }^{*}$ )

Raccogliendo si ha che:

$$
\operatorname{tg} \theta=-\frac{\tau}{\rho}
$$

$$
\frac{d y}{d s}=\frac{z}{r}, \frac{d z}{d s}=-\frac{x}{p}-\frac{y}{r}
$$

sono le condizioni necessarie e sufficienti affinche it punto $M_{1}(x y z)$ descriva una curva $C_{\mathrm{s}}$ trasformata di Combescure di $C ; C_{\mathrm{t}}$ avrà la tangente, la binormale, la normale principale e la retta rettificante parallele aile rette analoghe di $C$ ed avrà per equazioni intrinseche

$$
\rho_{s}=\rho f(s), \quad r_{\mathrm{s}}=r f(s), \quad \delta s_{s}=f(s) d s,
$$

*) Cfr. E. Cesaro, loc. eit., pag. 137. 
ove

$$
f(s)=\frac{\delta x}{d s}=\frac{d x}{d s}-\frac{z}{\rho}+1 .
$$

Pongo invece $\delta x=\delta z=0$ nelle (I). Allora il punto $M_{\mathrm{s}}$ si muoverà descrivendo una curva $C_{\mathrm{s}}$ riferita a $C$ per modo che la tangente a $C_{x}$ in $M_{\mathrm{x}}$ sarà parallela alla binormale di $C$ nel punto corrispondente $M$. Dirò che $C_{1}$ si deduce da $C$ con una trasformazione $B$ o che $C_{1}$ è una trasformata $B$ di $C$.

Mediante le (I) e (2) si possono dedurre direttamente le proprietà della trasformata $C_{1}$, ma si possono anche dedurre subito da un notevole teorema del BranchI ${ }^{*}$ ): per ogni curva $C$ ne esiste un'altra $C_{\mathrm{I}}$ (definita a meno di una traslazione) corrispondente per eguaglianza d'arco alla $C$; le due curvature e le direzioni della tangente e della binormale vengono per la trasformazione permutate. Precisamente: scelto per verso positivo della tangente di $C_{3}$ quello della binormale di $C$, il verso positivo della binormale di $C_{\mathrm{r}}$ sarà quello della tangente di $C$, mentre che le normali principali saranno parallele ma coi sensi positivi opposti; sarà poi

$$
p_{\mathrm{s}}=-r, \quad r_{\mathrm{s}}=-\rho_{\text {. }}
$$

Detta $B_{\circ}$ questa particolare, ma fondamentale, trasformazione $B$, notando che tutte le trasformate $B$ di una curva si possono dedurre l'una dall'altra con trasformazioni di Combescure, si ha che: la piu generale trasformazione $B$ è il prodotto della $B_{\mathrm{o}}$ per la piu generale trasformazione di Combescure (o viceversa).

In simboli, detta $T$ una trasformazione di Combescure, si ha

$$
B=B_{0} . T=T B_{\circ} \text {. }
$$

$\mathrm{Da}$ quanto precede, si raccoglie che:

$$
\frac{d x}{d s}=\frac{z}{p}-\mathrm{I}, \frac{d z}{d s}=-\frac{x}{p}-\frac{y}{r}
$$

sono le condizioni necessarie e sufficienti affinche il punto $M_{\mathrm{r}}(x y z)$ descriva una curva $C_{\mathrm{x}}$ trasformata $B$ di $C ; C_{\mathrm{x}}$ avrà la tangente e la binormale parallele alla binormale e alla tangente di $C$ (e con versi positivi eguali) $e$ la normale principale parallela alla normale principale di $C$ (ma con senso positivo opposto), ed avrà per equazioni intrinseche

$$
\text { (7) } \quad \rho_{\mathrm{r}}=-r f(s), \quad r_{\mathrm{s}}=-\rho f(s), \quad \delta s_{\mathrm{r}}=f(s) d s \text {, }
$$

*) Loc. cit., vol. I, pag. 53 . 
ove

$$
f(s)=\frac{\delta y}{d s}=\frac{d y}{d s}-\frac{z}{r} .
$$

Nelle (I) poniamo infine $\delta x=\delta y=0$. Allora il punto $M_{1}(x y z)$ si muoverd descrivendo una curva $C_{\mathrm{r}}$ riferita a $C$ per modo, che la tangente in $M_{1}$ sara parallela alla normale principale di $C$ nel punto corrispondente $M$. Dirò che $C_{1}$ si deduce da $C$ con una trasformazione $N$ - che $C_{\mathrm{r}}$ è una trasformata $N$ di $C$.

Applicando le (2) alla direzione della tangente di $C_{1}$, cioè ponendo $\alpha=0, \beta=0, \gamma=\mathrm{I}$, si ha

onde, posto

$$
\frac{\delta \alpha}{d s}=-\frac{1}{\rho}, \frac{\delta \beta}{d s}=-\frac{1}{r}, \frac{\delta \gamma}{d s}=0,
$$

saranno

$$
\delta \sigma=\sqrt{\delta \alpha^{2}+\delta \beta^{2}+\delta \gamma^{2}}=\frac{\sqrt{\rho^{2}+r^{2}}}{\rho r} d s,
$$

$$
\xi=\frac{\delta \alpha}{\delta \sigma}=-\frac{r}{\sqrt{\rho^{2}+r^{2}}}, \quad n=\frac{\delta \beta}{\delta \sigma}=-\frac{\rho}{\sqrt{\rho^{2}+r^{2}}}, \quad \zeta=\frac{\delta \gamma}{\delta \sigma}=0
$$

i coseni direttori della normale principale di $C_{1}$ e quindi

$$
\lambda=-\frac{\rho}{\sqrt{\rho^{2}+r^{2}}}, \quad \mu=\frac{r}{\sqrt{\rho^{2}+r^{2}}}, \quad \nu=0
$$

quelli della binormale; e la flessione sard

Posto

$$
\frac{\mathrm{I}}{p_{\mathrm{I}}}=\frac{\delta \sigma}{\delta s_{1}}=\frac{d s}{\delta s_{\mathrm{I}}} \cdot \frac{\delta \sigma}{d s}=\frac{d s}{\delta s_{\mathrm{I}}} \cdot \frac{\sqrt{\rho^{2}+r^{2}}}{\rho r} .
$$

$$
\operatorname{tg} \theta=-\frac{r}{\rho}
$$

$\theta$ è l'inclinazione della retta rettificante di $C$ sulla tangente, e si ha

$$
\begin{array}{lll}
\xi=-\operatorname{sen} \theta, & n=\cos \theta, & \zeta=0, \\
\lambda=\cos \theta, & \mu=\operatorname{sen} \theta, & \nu=0,
\end{array}
$$

onde la binormale di $C_{\mathrm{r}}$ è parallela alla retta rettificante di $C$.

Applicando infine le (2) alla direzione $\lambda \mu \nu$, si ha

e però

$$
\frac{\delta \lambda}{d s}=-\operatorname{sen} \theta \frac{d \theta}{d s}, \quad \frac{\delta \mu}{d s}=\cos \theta \frac{d \theta}{d s}, \quad \frac{\delta \nu}{d s}=0,
$$

$$
\frac{\mathrm{I}}{r_{\mathrm{I}}}=\sqrt{\left(\frac{\delta \lambda}{\delta s_{\mathrm{I}}}\right)^{2}+\left(\frac{\delta \mu}{\delta s_{1}}\right)^{2}+\left(\frac{\delta v}{\delta s_{\mathrm{I}}}\right)^{2}}=\frac{d s}{\delta s_{\mathrm{s}}} \cdot \frac{d \theta}{d s} .
$$


Raccogliendo si ba che:

$$
\frac{d x}{d s}=\frac{z}{p}-\mathrm{I}, \frac{d y}{d s}=\frac{z}{r}
$$

sono le condizioni necessarie e sufficienti affinche il punto $M_{1}(x y z)$ descriva una curva $C_{\mathrm{I}}$ trasformata $N$ di $C$; la tangente e la binormale di $C_{1}$ sono parallele alla normale principale ed alla retta rettificante di $C$; le equazioni intrinseche di $C_{\mathrm{r}}$ sono

ove

$$
\frac{f(s)}{p_{\mathrm{I}}}=\frac{\sqrt{\rho^{2}+r^{2}}}{\rho r}, \frac{f(s)}{r_{\mathrm{t}}}=\frac{d \theta}{d s}, \quad \delta s_{\mathrm{s}}=f(s) d s
$$

$$
f(s)=\frac{\delta z}{d s}=\frac{d z}{d s}+\frac{x}{p}+\frac{y}{r} .
$$

Ogni curva ha infinite trasformate $T B N$, perchè, in ciascuna trasformazione, delle 4 funzioni $x y z f$ di $s$ una è arbitraria. Specializzando convenientemente una di queste funzioni farò alcune applicazioni dei risultati precedenti, ma è chiaro che queste applicazioni possono protrarsi indefinitamente. Tralascerò le applicazioni già fatte dal Prof. Bianchi e che ho ricordate in principio.

I. Per $x=0$, le (6) dàno $z=\rho, y=-r \frac{d p}{d s}$, quindi $M_{1}(x y z)$ è un punto dell'asse del circolo osculatore di $C$ e questo asse è la tangente in $M_{1}$ alla $C_{1}$. Dunque, tenendo presenti le $(7)$ e (8) si ha che: gli assi dei circoli osculatori di una curva $C$ formano una superficie sviluppabile (sviluppabile polare di $C$ ) che è l'inviluppo dei suoi piani normali; lo spigolo di regresso $C_{1}$ (luogo dei centri delle sfere osculatrici di $C$ ) $\dot{e}$ una trasformata $B$ di $C$ ed ba per equazioni intrinseche le (7), ove

$$
-f(s)=\frac{\rho}{r}+\frac{d}{d s}\left(r \frac{d \rho}{d s}\right) \text {. }
$$

Se $p$ è costante, e solo allora, si ha $y=0$; in tal caso $M_{1}$ ha per coordinate $(o, o, \rho)$ e $C_{\mathrm{t}}$ è il luogo dei centri dei circoli osculatori di $C$; poi

$$
f(s)=-\frac{p}{r}, \quad p_{1}=p, r r_{1}=p^{2} .
$$

Si ha cosi il teorema di BouQuet che caratterizza $\mathrm{j}$ circoli storti: il luogo dei centri di curvatura di un circolo storto $C$ è anche un circolo storto $C_{\mathrm{r}} ; C_{\text {e }} C_{\mathrm{x}}$ sono l'uno il luogo dei centri dei circoli osculatori dell'altro e l'altro dell'uno, e si deducono l'uno dall'altro con una trasforma- 
zione $B$; banno egual flessione $e$ il prodotto delle loro torsioni eguaglia il quadrato della fiessione comune.

Se $f(s)=0$, e solo allora, si ha $p_{\mathrm{x}}=r_{\mathrm{s}}=0$, e quindi $C_{\mathrm{r}}$ si riduce ad un punto; poi

$$
M M_{1}^{2}=x^{2}+y^{2}+z^{2}=\rho^{2}+\left(r \frac{d \rho}{d s}\right)^{2},
$$

ma da $f(s)=$ o segue che $\rho^{2}+\left(r \frac{d \rho}{d s}\right)^{2}$ è costante, dunque $C$ è una curva sferica. Si ha cosi il teorema noto: $\frac{\rho}{r}+\frac{d}{d s}\left(r \frac{d \rho}{d s}\right)=0$ e la condizione necessaria e sufficiente affincbè una curva sia tracciata sopra una sfera di raggio $\sqrt{\rho^{2}+\left(r \frac{d \rho}{d s}\right)^{2}}$.

II. Pongo più generalmente $x=c$ (costante). Allora, per le (6),

$$
y=-r \frac{d \rho}{d s}-c \frac{r}{p}, \quad z=p
$$

e il teorema precedente si generalizza nel seguente: nel piano condotto per l'asse del circolo osculatore di una curva e parallelo al piano retificante, le rette parallele a questo asse e rigidamente legato ad esso generanu superficie sviluppabili $i$ cui spigoli di regresso $C_{\mathrm{x}}$ sono trasformate $B$ della curva ed hanno per equazioni intrinseche le (7), ove

$$
-f(s)=\frac{\rho}{r}+\frac{d}{d s}\left(r \frac{d \rho}{d s}\right)+c \frac{d}{d s}\left(\frac{r}{\rho}\right) .
$$

Queste curve $C_{\varepsilon}$ sono tuite trasformate $T$ dello spigolo di regresso della sviluppabile polare di $C$; sono congruenti con esso solo quando $C \dot{e} u n^{3} e-$ lica cilindrica, chè allora $\frac{d}{d s}\left(\frac{r}{p}\right)=0$.

III. Le (6) non sono soddisfatte per $x=z=0$ ossia le binormali di una curva non formano mai una superficie sviluppabile.

IV. Volendo invertire il problema I, cioè volendo trovare tutte le curve che banno una curva assegnata $C$ come luogo dei centri delle sfere osculatrici, basta porre $y=0$. Le (6) dànno

$$
\frac{d x}{d s}=\frac{z}{\rho}-\mathrm{r}, \frac{d z}{d s}=-\frac{x}{\rho}
$$

fra le quali eliminando $z$,

$$
\rho \frac{d^{2} x}{d s^{2}}+\frac{d \rho}{d s} \cdot \frac{d x}{d s}+\frac{x}{\rho}+\frac{d \rho}{d s}=0,
$$


ossia

ove si è posto

$$
\frac{d^{2} x}{d \varphi^{2}}+x+\frac{d \rho}{d \varphi}=0
$$

ne segue integrando

$$
\varphi=\int_{0}^{s} \frac{d s}{\rho}
$$

(12) $\left\{\begin{array}{l}x=c \operatorname{sen} \varphi+c^{\prime} \cos \varphi-\operatorname{sen} \varphi \int \operatorname{sen} \varphi d s-\cos \varphi \int \cos \varphi d s \\ z=c \cos \varphi-c^{\prime} \operatorname{sen} \varphi-\cos \varphi \int \operatorname{sen} \varphi d s+\operatorname{sen} \varphi \int \cos \varphi d s\end{array}\right.$

con $c$ e $c^{\prime}$ costanti arbitrarie.

Le (12) risolvono il problema proposto e quindi anche l'altro equivalente: trovare tutte le traiellorie ortogonali ad una semplice infinita di piani (i piani osculatori di $C$ ). Il problema, gid risoluto per altre vie, si risolve dunque mediante tre quadrature.

Le equazioni intrinseche di dette traiettorie sono

$$
p_{1}=z, \quad r_{1}=z \frac{\rho}{r}, \quad \delta s_{1}=\frac{z}{r} d s .
$$

V. Passando alla trasformazione $T$, pongo $x=0$ nelle (3); ne risulta

ed eliminando $z$,

$$
\frac{d y}{d s}=\frac{z}{r}, \frac{d z}{d s}=-\frac{y}{r} \text {, }
$$

ossia

$$
r \frac{d^{2} y}{d s^{2}}+\frac{d r}{d s} \cdot \frac{d y}{d s}+\frac{y}{r}
$$

ove si è posto

$$
\frac{d^{2} y}{d \psi}+y=0
$$

$$
\psi=\int_{0}^{s} \frac{d s}{r} ;
$$

ne segue integrando

$$
\left\{\begin{array}{l}
y=c \cos \psi+c^{\prime} \operatorname{sen} \psi \\
z=c^{\prime} \cos \psi-c \operatorname{sen} \psi
\end{array}\right.
$$

con $c$ e $c^{\prime}$ costanti arbitrarie.

Condotte per $M$ le due normali di $C$ di inclinazione $\psi$ e $\frac{\Pi}{2}+\psi$ sulla normale principale, è chiaro che il punto $M_{x}(0, y, z)$ definito dalle (I4) è quel punto del piano normale di $C$ che dista di $c$ e $c$ dalle suddette normali. Or se si tien presente che la (1 3 ) è la condizione neces-

Rend. Circ. Matem. Palermo, t. XX (1905). - Stampato il Is maggio Igos. 
saria e sufficiente affinchè l'una e (quindi) l'altra normale generino superficie sviluppabili ${ }^{*}$ ), si può asserire che: un punto del piano normale di una curva $C$, invariabilmente legato a due normali oriogonali tra luro, genera una curva $C_{1}$ trasformata $T$ di $C$, quando una delle due normali (e quindi l'altra) genera una superficie sviluppabile.

Osservando poi che le curve $C_{\mathrm{r}}$ sono traiettorie ortogonali ai piani normali di $C$, si ha che le (I4) risolvono il problema di: costruire le traiettorie ortugonali ad una semplice infinità di piani, quando è nota una di essa. Questo problema dunque si risolve mediante una sula quadratura, la (I 3), mentre che il problema generale richiede tre quadrature. (Cfr. IV).

Essendo poi

$$
M M_{1}^{2}=x^{2}+y^{2}+z^{2}=c^{2}+c^{\prime 2},
$$

ne segue che: due traiettorie ortogonali ad una semplice infinità di piani sono dappertutto equidistanti.

VI. Ponendo $z=0$, le (3) dànno $y=c$ (costante) e $x=-\frac{p}{r} c$, e però $M_{\mathrm{r}}(x y z)$ sta sulla retta rettificante di $C$ in $M$. Si ha cosi il teorema di CEsÀro ${ }^{* *}$ ) le rette parallele alla tangente di una curva $C$, contenute nel piano rettificante e rigidamente legate al suo triedro fondamentale, generano superficie sviluppabili.

Qui possiamo aggiungere che: gli spigoli di regresso $C_{1}^{\prime}$ sono trasformate $T$ di $C$; le loro equazioni intrinseche sono

$$
s_{\mathrm{I}}=s-\frac{\rho}{r} c, \quad p_{\mathrm{s}}=\rho\left[\mathrm{I}-c \frac{d}{d s}\left(\frac{\rho}{r}\right)\right], r_{\mathrm{z}}=r\left[\mathrm{I}-c \frac{d}{d s}\left(\frac{\rho}{r}\right)\right],
$$

ed banno in comune con $C$ la sviluppabile rettificante.

Una curva è geodetica della sua sviluppabile rettificante, dunque $C$ e $C_{1}$ sono geodetiche della comune sviluppabile rettificante ed egualmente inclinate sulle generatrici; poi $M M_{1}=c \sqrt{\mathrm{I}+\left(\frac{\rho}{r}\right)^{2}}$.

Dunque: se a partire da ciascun punto $M$ di una geodetica $C$ di una superficie sviluppabile si porta sulla corrispondente generatrice il segmento $M M_{\mathrm{I}}=c \sqrt{\mathrm{I}+\left(\frac{p}{r}\right)^{2}}$, il luogo del punto $M_{\mathrm{I}} \dot{e}$ un'altra geodetica egualmente inclinata sulla generatrice.

*) Cesáro, loc. cit., pag. I 39.

**) Loc. cit., pag. I 49 . 
Se $C$ è un'elica cilindrica, cioè se $\frac{p}{r}$ è costante, si ha

$$
s_{1}=s+\text { cost., } \quad p_{1}=p_{,}, r_{1}=r,
$$

e però il teorema evidente: un'elica cilindrica pùo scorrere sul cilindro nel senso delle generatrici e senza deformarsi, e del quale il precedente può ritenersi come una generalizzazione.

VII. Per $y= \pm p$, le (3) dàno

$$
z= \pm r \frac{d \rho}{d s}, \quad x=\mp \rho\left[\frac{\rho}{r}+\frac{d}{d s}\left(r \frac{d \rho}{d s}\right)\right] \text {. }
$$

Or, come è facile vedere, il punto $P\left(y= \pm p, z= \pm r \frac{d p}{d s}\right)$ del piano normale di $C$ in $M$ non è altro che il centro della sfera osculatrice (Cfr. I) $\left(y=-r \frac{d p}{d s}, z=p\right)$ rotato dell'angolo $\pm \frac{\pi}{2}$ nel piano stesso intorno ad $M$; dunque: la parallela alla tangente in un punlo $M$ di una curva $C$ condotta pel centro della sfera osculatrice, rotato dell'angolo $\pm \frac{\pi}{2}$ intorno ad $M$ nel piano normale, genera una superficie sviluppabile, quando $M$ si muove sulla curva.

Se $C$ è una curva sferica, risulta (Cfr. I) $x=0$ e però : il centro di una sfera rotato di un angolo retto intorno ad un punto $M$ di una curva tracciata su di essa, nel piano normale di questa, genera una trasformata $T$ della curva, quando $M$ si muove sulla curva.

VIII. Le (9) non son soddisfatte da $x=y=0$ ossia le normali principali di una curva non formano mai una superficie sviluppabile.

IX. Dalla ( 10 ) segue che $\frac{\mathrm{I}}{r_{\mathrm{r}}}=0$ solo quando $\theta$ è costante, ossia quando $\frac{p}{r}$ è costante; dunque: fra le curve storte le eliche cilindriche sono caratterizzate dal fatto che tutte le loro trasformate $N$ sono curve piane contenute in piani perpendicolari alle generatrici del cilindro.

$\mathrm{X}$. Per $y=0$ le (9) dánno $z=0, x=c-s$ con $c$ costante arbitraria. Allora $M_{1}(x y z)$ è un punto della tangente in $M$, ed essendo $M M_{\mathrm{r}}=x=c-s$, il luogo $C_{\mathrm{r}}$ dei punti $M_{\mathrm{r}}$ è una sviluppante di $C$; dunque: le sviluppanti di una curva sono trasformate $N$ di essa.

Più generalmente, per $y=c^{\prime}$ (costante) le (9) dànno pure $z=0$, $x=c-s$; quindi: un punto di una sviluppante di una curva, spostato di un segmento costante $c$ nel verso della binormale nel punto corrispondente della curva, genera una trasformata $N$ della curva. 
XI. Ponendo nelle (9) $x=0$, si ha $y=\int \frac{p}{r} d s, z=\rho$. La curva $C_{\mathrm{t}}$ descritta dal punto $M_{\mathrm{I}}(x y z)$ giace sulla sviluppabile polare di $C$, e su questa è una traiettoria ortogonale delle generatrici, chè la tangente in $M_{1}$, essendo parallela alla normale principale di $C$ in $M$, è perpendicolare all'asse del circolo osculatore. Dunque: la normale principale di una curva, spostata parallelamente a sè stessa nel piano normale di $y=\int \frac{p}{r} d s$, descrive una superficie sviluppabile, il cui spigolo di regresso giace sulla sviluppabile polare della curva e ne incontra le generatrici ad angolo retto.

Il punto $P\left(0, \int \frac{p}{r} d s, p\right)$ dell'asse polare di una curva è dunque tale che la parallela per esso alla normale principale e l'asse polare stesso generano superficie sviluppabili. Ma i valori $y=\int \frac{p}{r} d s, r=p$ soddisfanno anche le (3) e dàno

$$
x=-\rho\left(\frac{d \rho}{d s}+\frac{\mathbf{I}}{r} \int \frac{\rho}{r} d s\right),
$$

quindi anche la parallela alla tangente condotta per $P$ genera una superficie sviluppabile. Dunque: le parallele alla tangente alla binormale $e$ alla normale principale di una curva, condotte pel punto del piano normale distante per $\rho$ e $\int \frac{\rho}{r} d s$ dalle ultime due rette, generano tre superficie sviluppabili, $i$ cui spigoli di regresso sono trasformate $T B N$ della curva.

Torino, febbrajo I905.

Gustavo Sannia. 